\title{
Temperature Profiles of Window Jet Plume
}

\author{
Jun-ichi Yamaguchi ${ }^{1}$ and Takeyoshi Tanaka ${ }^{2}$ \\ ${ }^{1}$ Technical Research Institute, Obayashi Corporation, Japan \\ ${ }^{2}$ DPRI, Kyoto University, Japan
}

\begin{abstract}
In order to develop a means to assess the effectiveness of some design elements for preventing upper floor fire spread, such as window geometry and arrangement of eaves above windows, possibility of scaling of window jet plume temperature distribution by means of a nondimensional temperature was explored. Experiments were conducted using two geometrically similar setups having different sizes to investigate the applicability of the non-dimensional temperature to various conditions.

It was found that the non-dimensional temperature is independent of size and fire temperature but is uniquely determined only by geometrical conditions, that is, it is possible to predict real scale window jet plume temperatures based on the results of reduced scale experiments.
\end{abstract}

Keywords : Window jet plume, Upper floor fire spread, Non-dimensional temperature

\section{INTRODUCTION}

Show in Figure 1, window flames or window jet plumes are a major cause of upper floor fire spread and perhaps not a negligible cause of fire spread to adjacent buildings in densely built urban area. It is a common practice to assess the hazard of upper floor fire spread based on the estimation of axis temperature of window jet plume or the height of the window flame [1,2]. Ohmiya et al. found that the predicting method of the axis temperature taking into consideration excess fuel gas ejected from the fire room windows [3]. And Yamada et al. make a model that the predicting the flame height of window jets with eaves or balconies upper the window [4].
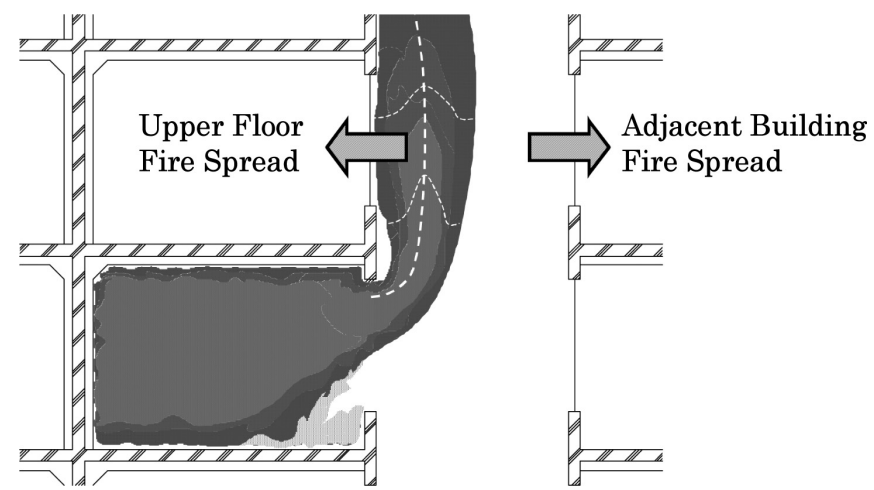

Figure 1 Upper Floor Fire Spread and Adjacent Building Fire Spread 
The most complete study on window flames and plumes was conducted by Yokoi. He found the non-dimensional parameter for scaling the axis temperature of the window flames, which gives us a means to predict the axis temperature under various window geometry and fire room temperature [1]. However, the temperature which actually affects to fire spread is not exactly the axis temperature but the temperature to which windows on upper floors are directly exposed. Since the window jet plume temperature is the highest at its axis, it will require excessive, though conservative, fire resistant performance to window panes if the breaking of the window is assessed based on the axis temperature. Generally speaking, the conditions of the usual preventive measures of upper floor fire spread, such as geometry of window, spandrel or balcony differs from one building to another. The only accurate way to investigate the preventive effects of these factors on upper floor fire spread is to conduct the experiments under the conditions reflecting the real situation. However, conducting full-scale experiments are not always affordable. Hence, a practical means to take into account such design features in assessing the hazard of fire spread by window flames may be to use reduced scale experiments provided that an appropriate scaling parameter is established.

\section{NON-DIMENSIONAL TEMPERATURE}

Yokoi found that the temperature along the axis of window plume can be scaled by the non-dimensional temperature, defined by

$$
\Theta=\frac{\Delta T_{0} r_{0}^{5 / 3}}{\left(\frac{T_{\infty} Q_{D}^{2}}{C_{p}^{2} \rho^{2} g}\right)^{1 / 3}}
$$

where $\Delta T_{0}$ is the temperature rise at arbitrary position along the plume axis, $r_{0}$ is the equivalent radius of the window jet, $T_{\infty}$ is the ambient temperature, $Q_{D}$ is the heat ejected along with the window jet, $C_{p}$ is the specific heat of air, $\rho$ is the gas density at arbitrary position along the plume axis and $g$ is the acceleration due to gravity.

Yokoi's non-dimensional temperature can be transformed into a form more popular in the current fire research community, i.e.

$$
\Theta \equiv\left(\frac{\Delta T_{0} / T_{\infty}}{Q_{D\left(r_{0}\right)}^{*}{ }^{2 / 3}}\right) /\left(\frac{T_{0}}{T_{\infty}}\right)^{2 / 3}
$$

where $T_{0}$ is the temperature at arbitrary position along the plume axis, i.e. $T_{0}=\Delta T_{0}+T_{\infty}$, and $Q_{D\left(r_{0}\right)}^{*}$ is the non-dimensional heat flow rate of window jet define as

$$
Q_{D\left(r_{0}\right)}^{*}=\frac{Q_{D}}{C_{p} \rho_{\infty} T_{\infty} \sqrt{g} r_{0}^{5 / 2}}
$$

where $\rho_{\infty}$ is the ambient air density.

However, the Yokoi's non-dimensional temperature is considered to be valid for not 
only plume axis but also off-axis field. Therefore extending the temperature rise $\Delta T_{0}$ to the rise temperature at not only arbitrary position along the plume axis but arbitrary positions, $(x, y, z)$, the plume, i.e. $\Delta T(x, y, z)$, and neglecting $\left(T / T_{\infty}\right)^{2 / 3}$ in Equation (2). we employ

with

$$
\Theta\left(x^{*}, y^{*}, z^{*}\right) \equiv \frac{\Delta T(x, y, z) / T_{\infty}}{Q_{D\left(r_{0}\right)}^{*}{ }^{2 / 3}}
$$

$$
\left(x^{*}, y^{*}, z^{*}\right)=\left(\frac{x}{r_{0}}, \frac{y}{r_{0}}, \frac{z}{r_{0}}\right)
$$

as the scaling parameter for convenience in practical application for the window jet plume temperature.

Also, in stead of $Q_{D\left(r_{0}\right)}^{*}$ given by Equation (3), which uses equivalent radius $r_{0}$ as the representative length, we use $Q_{D}^{*}$ more conveniently defined using the height of window, $H$, as the representative length, namely,

$$
Q_{D(H)}^{*}=\frac{Q_{D}}{C_{p} \rho_{\infty} T_{\infty} \sqrt{g} H^{5 / 2}}
$$

since, the representative length do not necessarily have to be equivalent window jet radius, as long as similarity among different scales is maintained.

On the other hand, when the window aspect ratio (=the window width / the window height) is large, the window jet plume in near height from the window, may behave more as a plume form a line heat source than form a point heat source. In this case, it may be more appropriate to define the non-dimensional heat release rate of line heat source, i.e.

$$
Q^{\prime *}=\frac{Q^{\prime}}{C_{p} \rho_{\infty} T_{\infty} \sqrt{g} W^{3 / 2}}
$$

where $Q^{\prime}$ is the heat release rate per unit length at the line heat source and $W$ is the width of the line heat source.

The non-dimensional heat release rate of line heat source, $Q^{\prime *}$, is extended to the window jet plume. Using $Q^{\prime}=Q_{D} / B, W=H$ in Equation (6), we have the definition of $Q_{D}^{*}$ slightly different from Equation (5) as

$$
Q_{D(H)}^{\prime^{*}}=\frac{Q_{D}}{C_{p} \rho_{\infty} T_{\infty} \sqrt{g} B H^{3 / 2}}
$$

The non-dimensional temperatures hold in situation that the effect of the heat transfer from the window jet plume to surrounding walls is insignificant. 


\section{EXPERIMENTS}

\subsection{Model Fire Compartments}

The two geometrically similar experimental setups as shown in Figure 2 are used in this series of experiments. The temperatures of window jet plumes were measured at geometrically similar positions relative to the window height under various conditions of window geometry and fire size. The inside dimensions of the small scale and the middle scale cubic fire compartments are $0.5 \mathrm{~m}$ and $1.5 \mathrm{~m}$, respectively. The " $h "$ in Figure 2 is the interval length of thermocouple setup, $h=0.15 \mathrm{~m}$ for middle scale experiment and $h=0.05 \mathrm{~m}$ for small scale experiment. Methanol in the pans of different sizes are placed on the floor of the compartment and burned as the fire source. The size and geometry of the window of the compartment are changed using the panels having different windows.

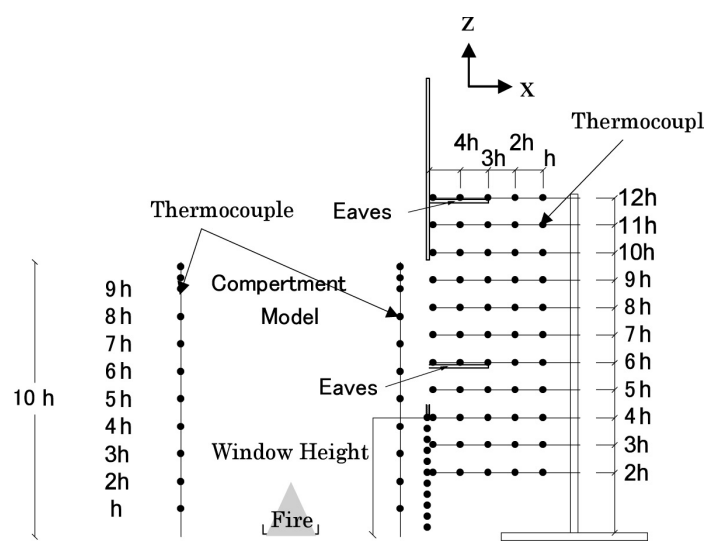

Section (Middle Scale Experiment : $h=0.15 \mathrm{~m}$, Small Scale Experiment $: h=0.05 \mathrm{~m}$ )

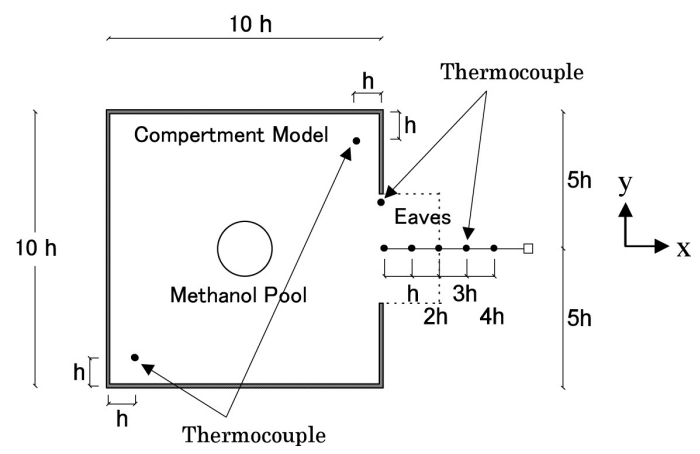

Plan(Middle Scale $: h=0.15 \mathrm{~m}$, Small Scale $: h=0.05 \mathrm{~m}$ )

Figure2 Schematic Diagram of Experimental Apparatus 


\subsection{Measurement}

The temperatures were measured in the compartment, at the window and outside the window. The locations of the thermocouples for the temperature measurements are shown in Figure 2. The temperatures at the compartment were measured by 9 thermocouples arrayed vertically with 15 and $5 \mathrm{~cm}$ spacing in the middle scale and the small scale setups, respectively. The temperatures at the window were measured by the thermocouples arrayed at $3 \mathrm{~cm}$ from one of the edges of window and with $3 \mathrm{~cm}$ and $1.5 \mathrm{~cm}$ spacing in the middle and the small scale setups, respectively. The temperatures outside the window were measured by 55 thermocouples arrayed vertically and horizontally with 15 and $5 \mathrm{~cm}$ spacing in the middle scale and the small scale setups, respectively.

\subsection{Experimental Conditions}

The conditions of the window, eaves and the fire source in the middle and small scale experiments are shown in Table $1(a)$ and $1(b)$, respectively. The experiments were conducted for every combination of these conditions. 
Table 1(a) Experimental Conditions for Middle Scale Experiment

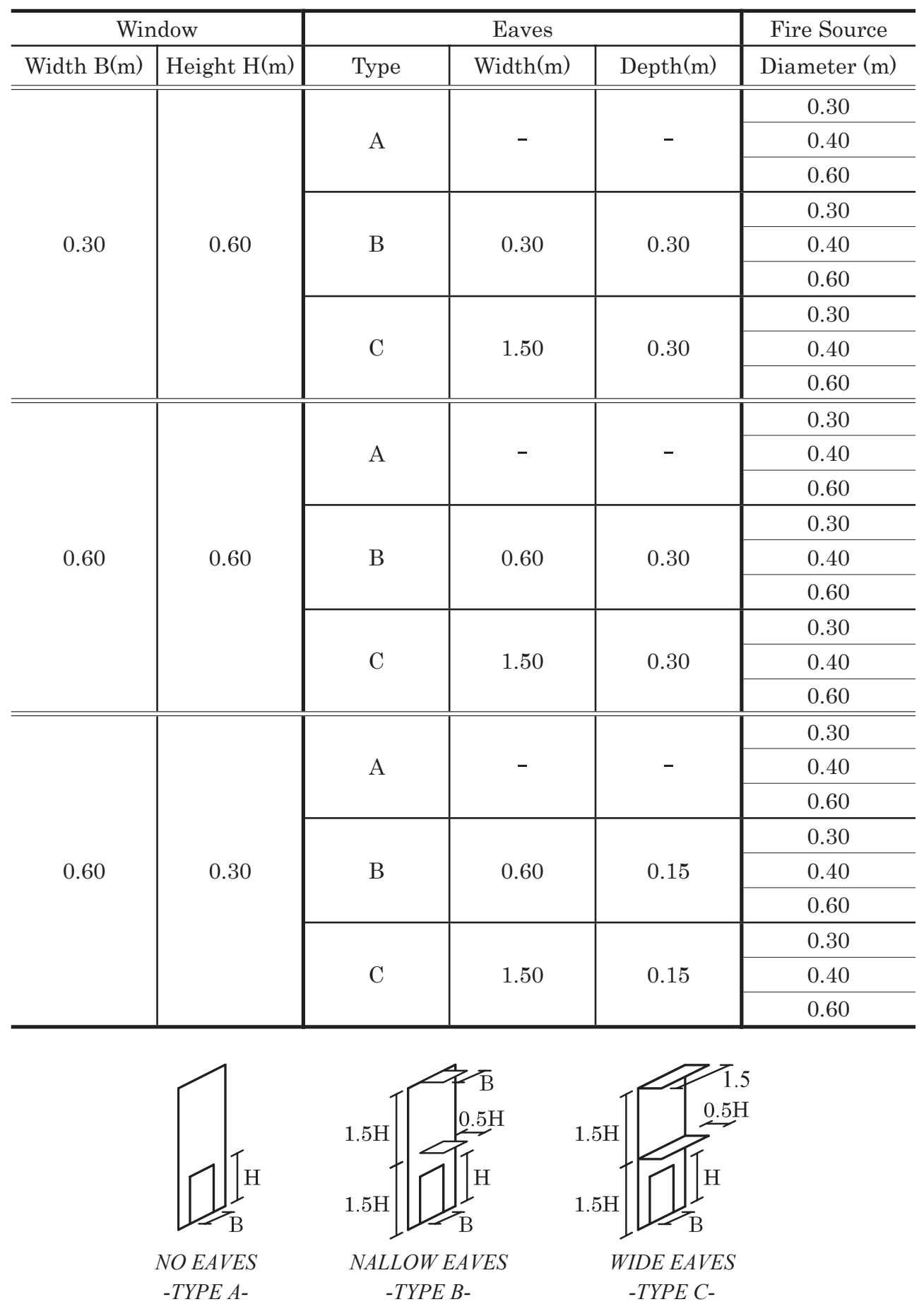


Table 1(b) Experimental Conditions for Small Scale Experiment

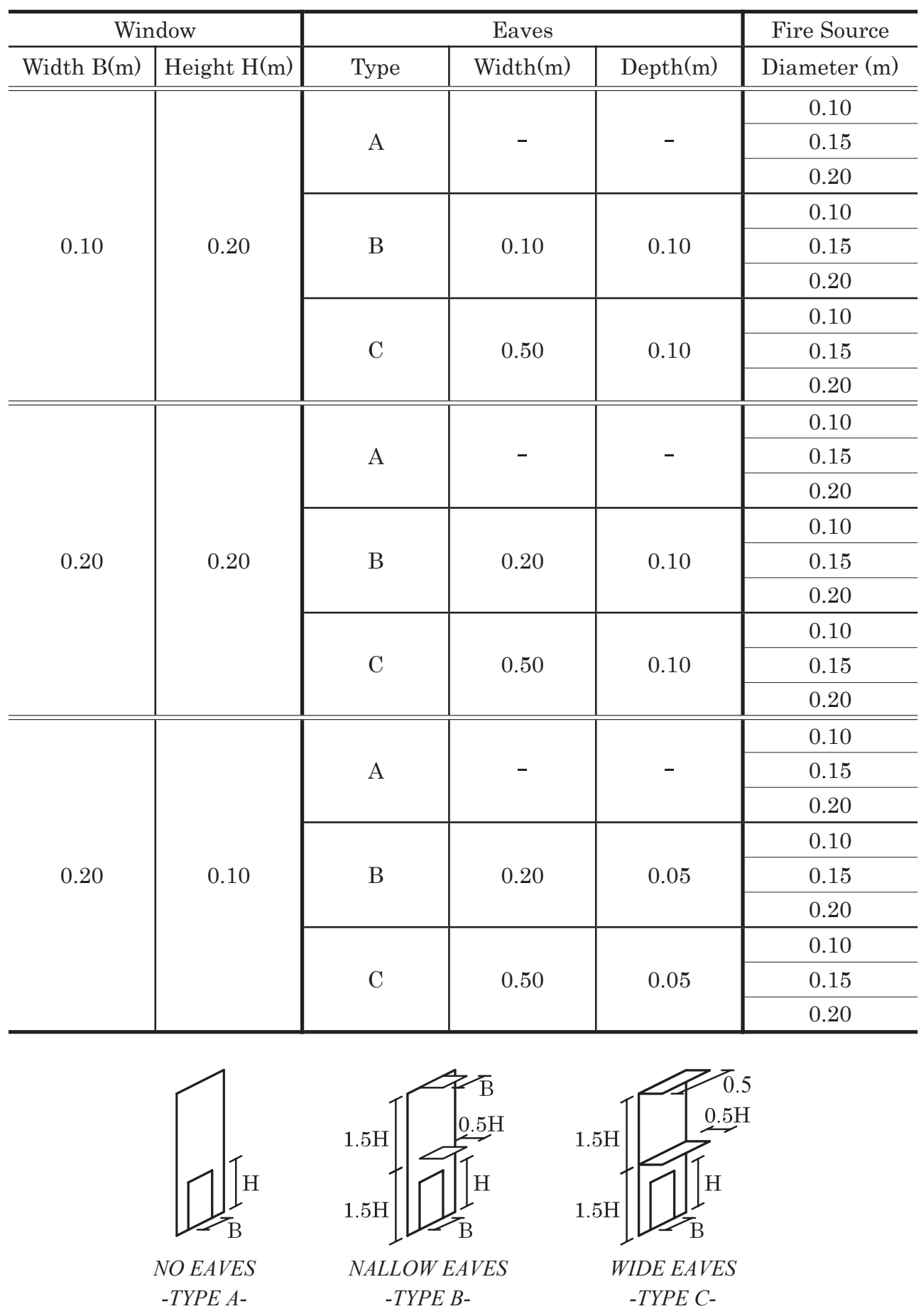




\section{ANALYSES OFTHE EXPERIMENTAL RESULTS}

\subsection{Temperature of the window jet plume}

Figure 3(a) shows the examples of the isotherms of the raw measured temperatures of the window jet plumes from the same window in the small-scale experiments but for different heat flow rates. On the other hand, Figure 3(b) shows the examples of the isotherms of the raw measured temperatures of the window jet plumes in the middle-scale experiment, but similar window geometry with the small-scale experiments. The difference in density in the isotherms indicates the difference in temperatures of the window jet, which naturally differ depending on the heat flow rate and the size of the windows. Note also that the isotherms of the small and the middle scale experiments are drawn in the flow fields with different scales.

Incidentally, the heat flow rate of window jet $Q_{D}$ in Equation (5) and Equation (7) was estimated as

$$
Q_{D}=C_{p} m_{D} \Delta T
$$

where $C_{p}$ is the specific heat of air at constant pressure, $\Delta T$ is the average temperature rise of window jet and $m_{D}$ is the mass flow rate of the window jet[5]. $m_{D}$ was calculated from the compartment temperature and the neutral plane height at the window,

$$
m_{D}=\frac{2}{3} C_{D} B\left\{2 g \rho_{s}\left(\rho_{\infty}-\rho_{s}\right)\right\}^{1 / 2}\left(H-Z_{n}\right)^{3 / 2}
$$

where $C_{D}$ is window flow coefficient, $B$ is the window width, $g$ is gravitational acceleration, $\rho_{s}$ is the window jet density, $\rho_{\infty}$ is the ambient air density, $H$ is the window height and $Z_{n}$ is the neutral plane height at the window. 

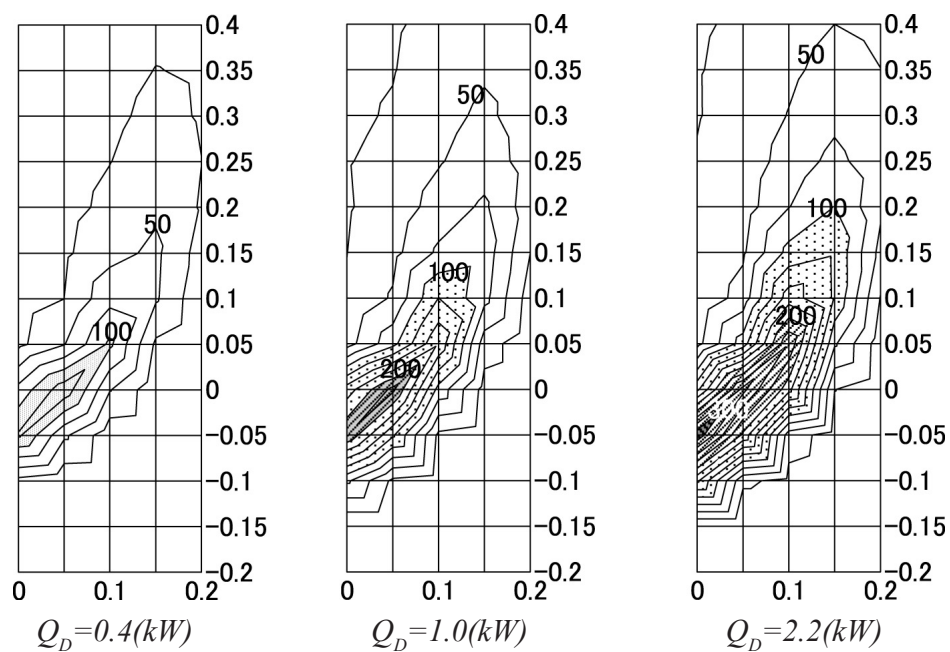

Window Shape $0.1(B) \times 0.2(H)(m)$

Figure 3(a) Raw Isotherms (Small Scale Experiment)
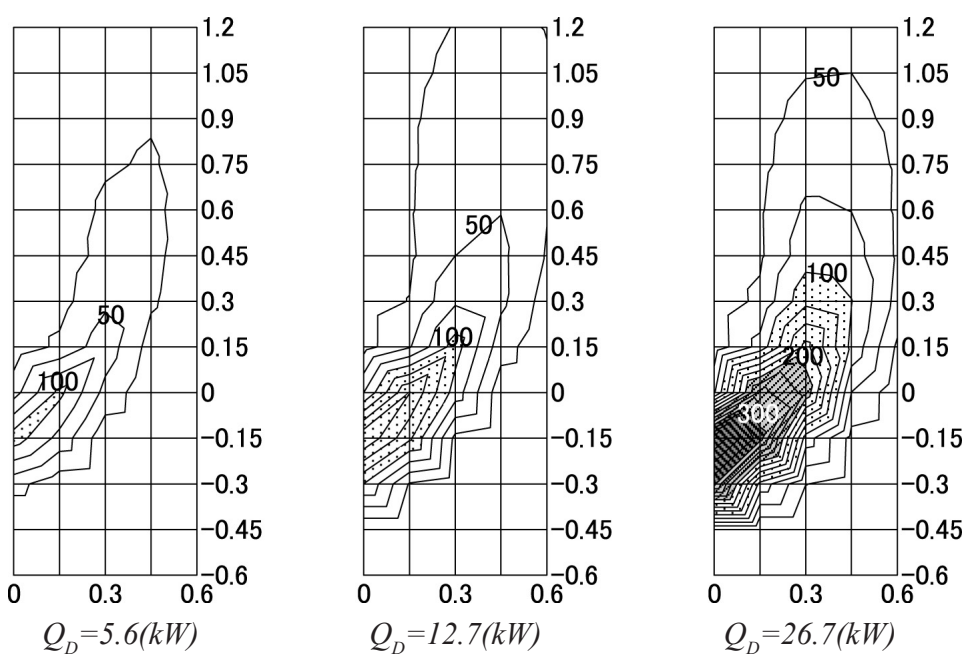

Window Shape 0.3(B) : 0.6(H) $(\mathrm{m})$

Figure 3(b) Raw Isotherms (Middle Scale Experiment)

On the other hand, Figure 4(a) and 4(b) shows the profiles of the non-dimensional temperatures, in which the measured raw temperatures in Figure 3(a) and 3(b) are processed by Equation (4) using Equation (5) as the non-dimensional heat flow rate. In order for non-dimensional temperatures in these figures to be in agreement, the window jet geometry, represented by the aspect ratio, $n\left(\equiv B /\left(\mathrm{H}-Z_{n}\right)\right)$, between must be similar the small scale experiments and the middle scale experiments. The aspect ratio, $n$, could not be kept the same in these experiments, as can be seen from the values indicated below the isotherms, because the neutral plane height, $Z_{n}$, is dependent on the room fire conditions. It can be seen that all the window jet plume isotherms, which differ in Figure 
3(a) and 3(b), have become to be almost the same regardless the difference in size and heat flow rate, in terms of the non-dimensional temperature. The reason for the slight difference, which is seen among the non-dimensional temperatures, is considered to be partly due to the slight difference of the window jet geometry.
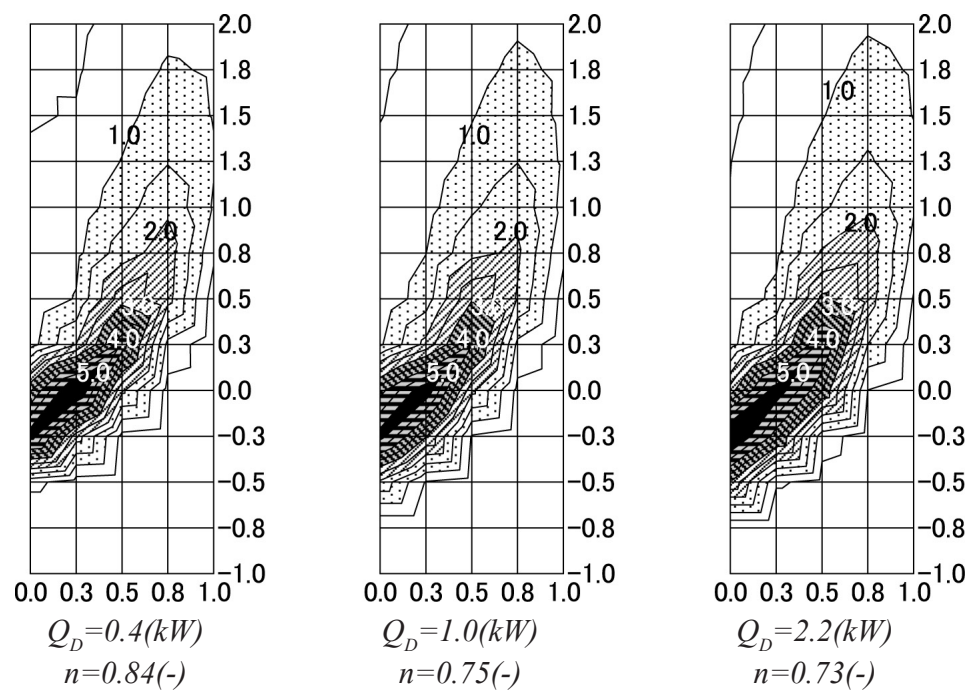

Figure 4(a) Non-dimentional Isotherms (Small Scale Experiment)
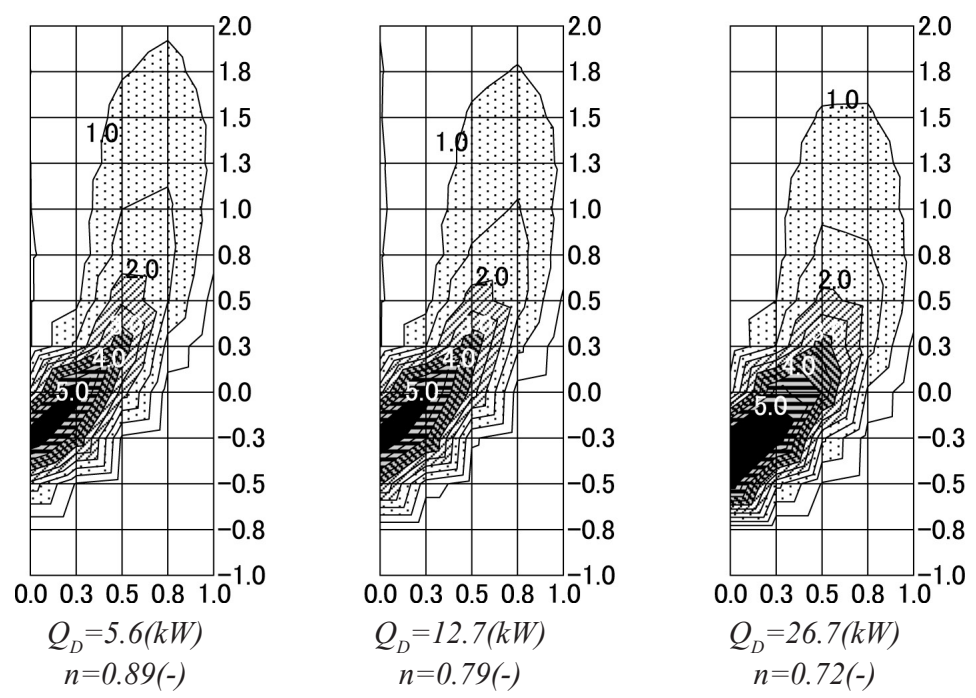

Figure 4(b) Non-dimentional Isotherms (Middle Scale Experiment)

Figure 5(a) and 5(b) shows the profiles of the non-dimensional temperatures processed by Equation (4) using the non-dimensional heat flow rate defined by Equation (7). It can be seen that all the window jet plume isotherms have become to be almost the same. If 
window jet section has the same aspect ratio, the non-dimensional temperature defined by Equation (4) mean physically the same parameter, whether the non-dimensional heat flow rate is defined by Equation (5) or Equation (7).
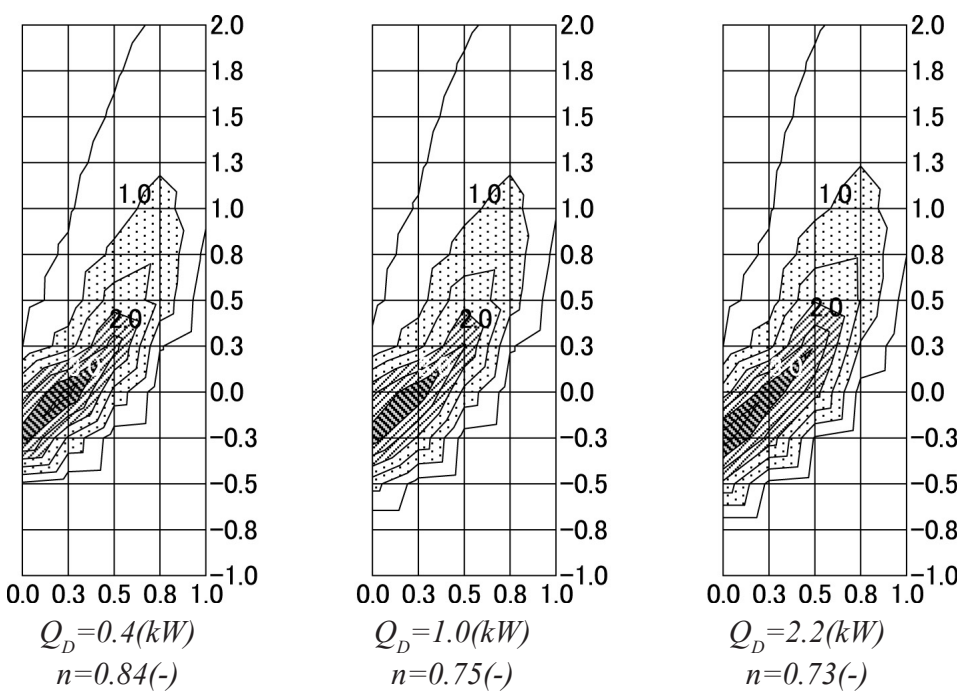

Figure 5(a) Non-dimentional Isotherms (Small Scale Experiment)
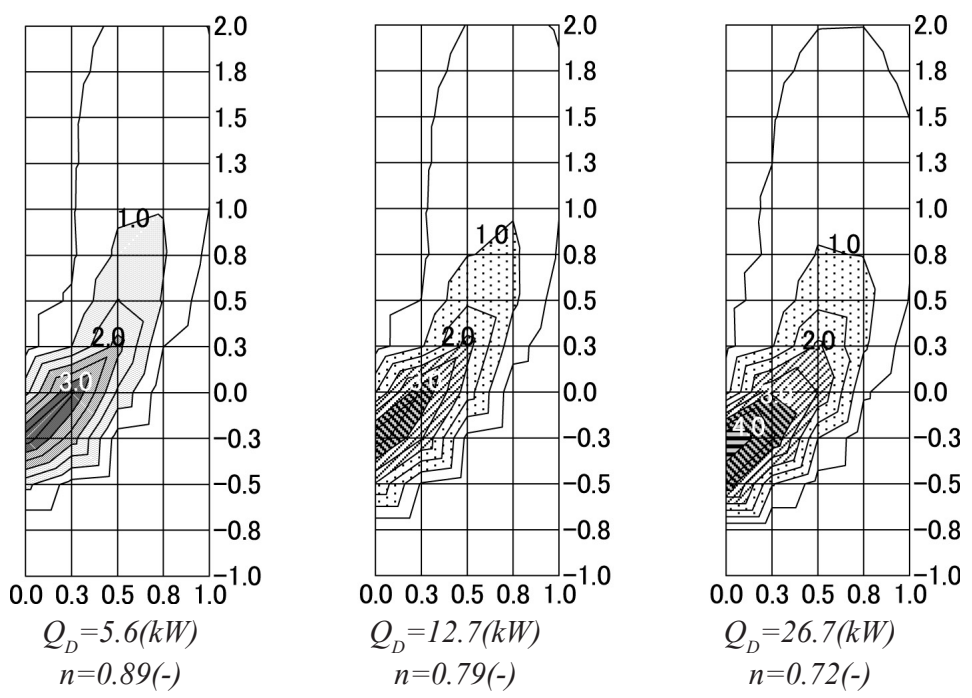

Figure 5(b) Non-dimentional Isotherms (Middle Scale Experiment)

Figure 6 shows examples of the isotherms of the raw measured temperatures of the window jet plume for different size and geometry of window. On the other hand, Figure 7 shows the profiles of the non-dimensional temperatures processed by Equation (7). It is demonstrated that the non-dimensional isotherms agree sufficiently well regardless the difference in size if the window jet geometry is similar. 


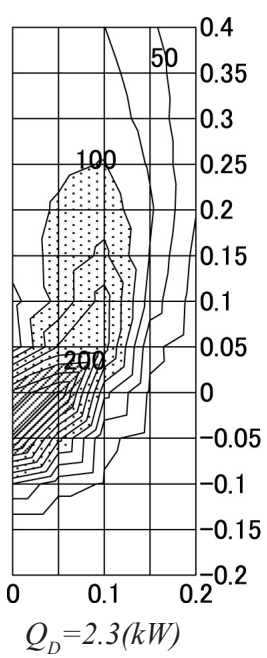

$n=1.72(-)$

Small Scale Exp.

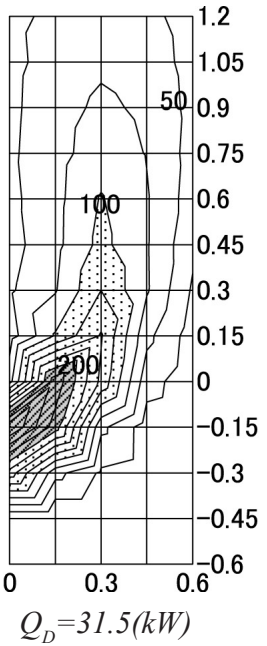

$n=1.68(-)$

Middle Scale Exp.

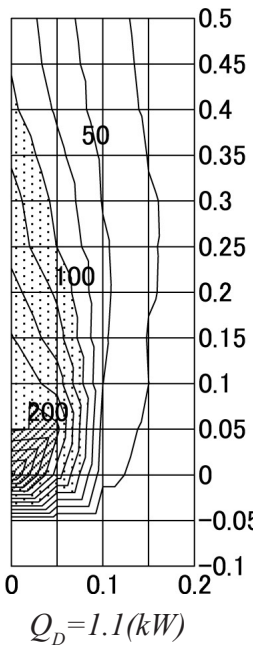

$n=2.84(-)$

Small Scale Exp.

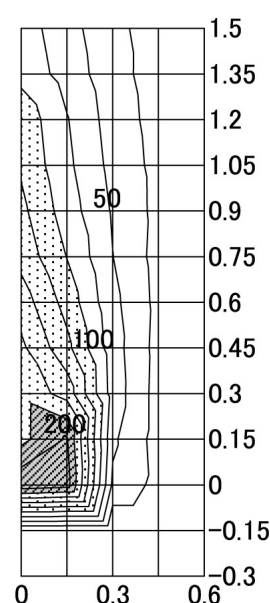

$Q_{D}=19.2(\mathrm{~kW})$

$n=2.99(-)$

Middle Scale Exp.

Figure 6 Raw Isotherms

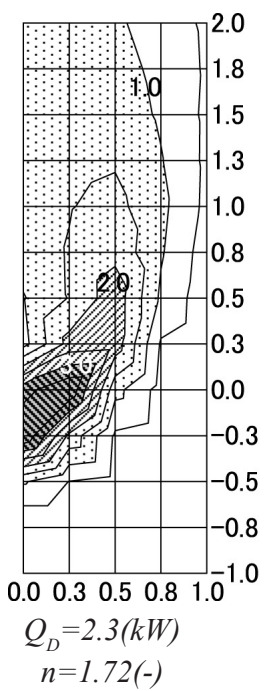

Small Scale Exp.

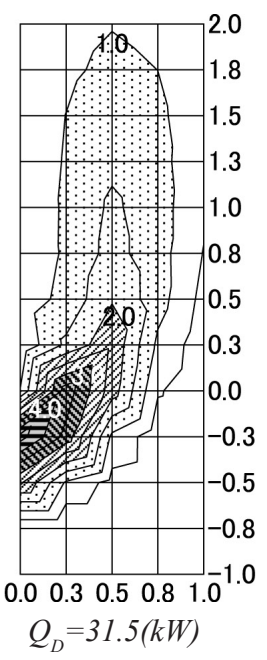

$n=1.68(-)$

Middle Scale Exp.

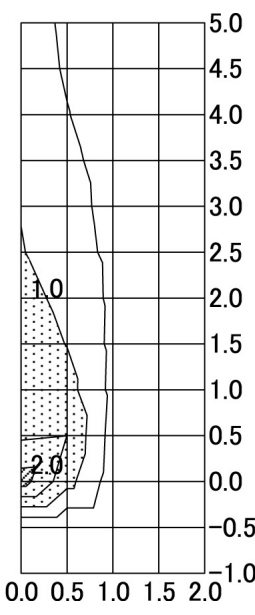

$Q_{D}=1.1(\mathrm{~kW})$

$n=2.84(-)$

Small Scale Exp.

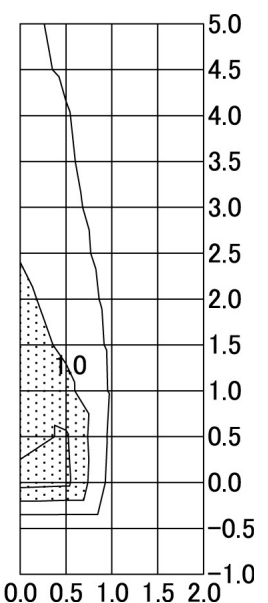

$Q_{D}=19.2(\mathrm{~kW})$

$n=2.99(-)$

Middle Scale Exp.

Figure 7 Non-dimentional Isotherms

Figure $8(a), 8(b), 9(a)$ and $9(b)$ shows examples of the non-dimensional temperature profiles of the window jet plumes from windows with eaves. The width of the narrow eaves is the same as the window width and that of the wide eaves is the same of the full compartment width. As the width of eaves becomes large, the window jet plume axis is kept more remote away from the surface of the wall. 

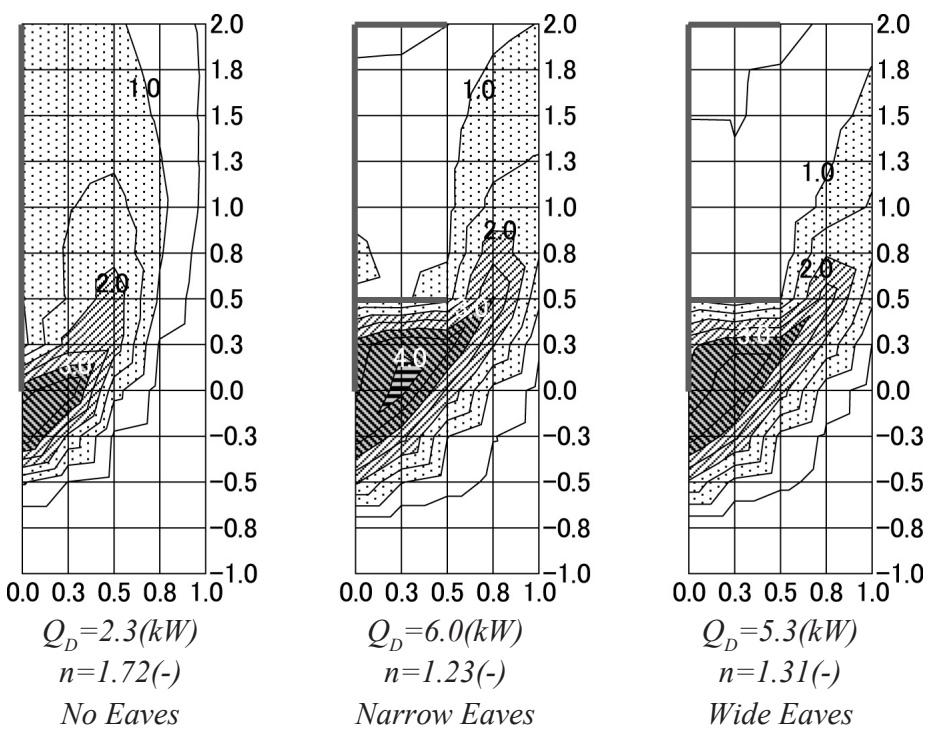

Window Shape $0.2(B) \times 0.2(H)(m)$

Figure 8(a) Non-dimentional Isotherms (Small Scale Experiment)

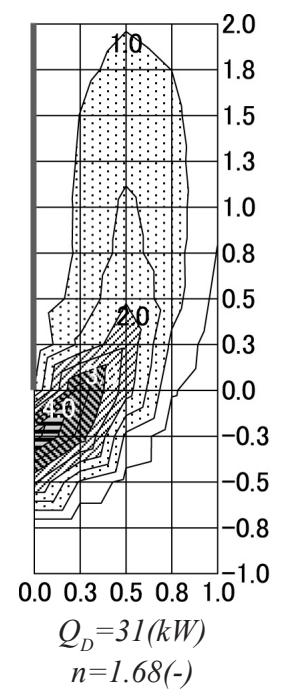

No Eaves

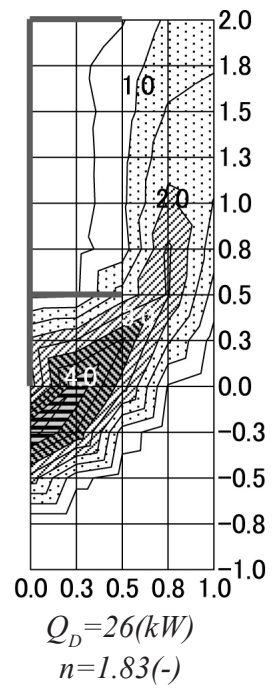

Narrow Eaves

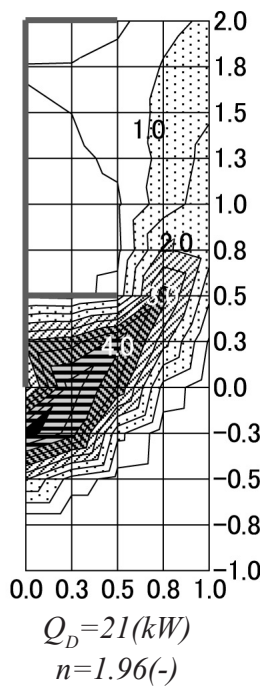

Wide Eaves

Window Shape $0.6(B) \times 0.6(H)(m)$

Figure 8(b) Non-dimentional Isotherms (Middle Scale Experiment) 


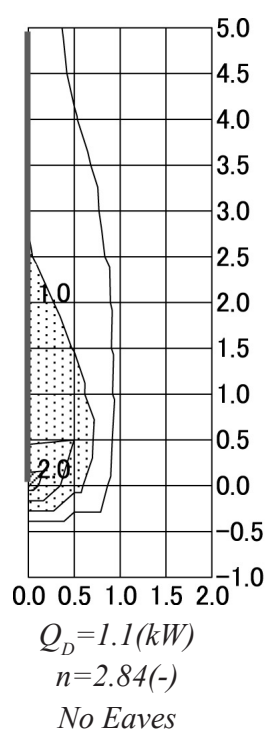

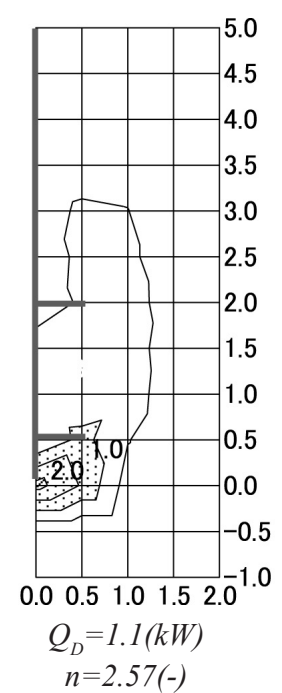

Narrow Eaves

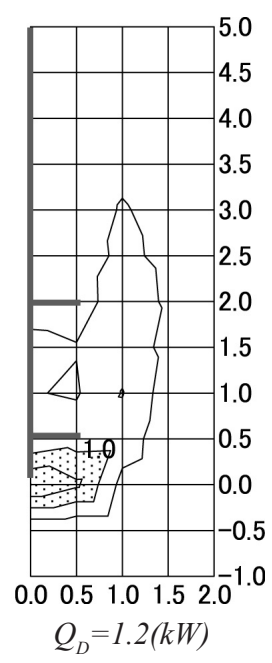

$n=2.54$ (-)

Wide Eaves

Window Shape $0.2(B) \times 0.1(H)(m)$

Figure 9(a) Non-dimentional Isotherms (Small Scale Experiment)

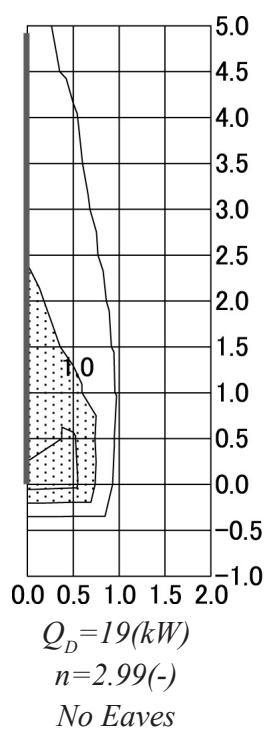

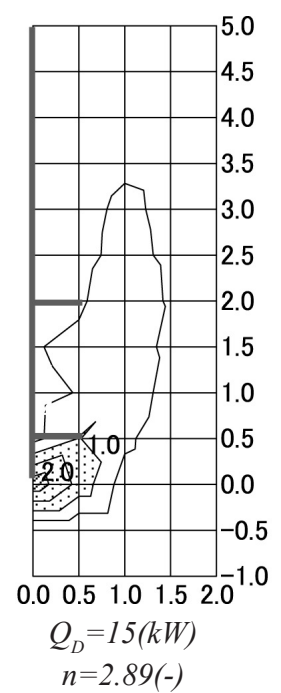

Narrow Eaves

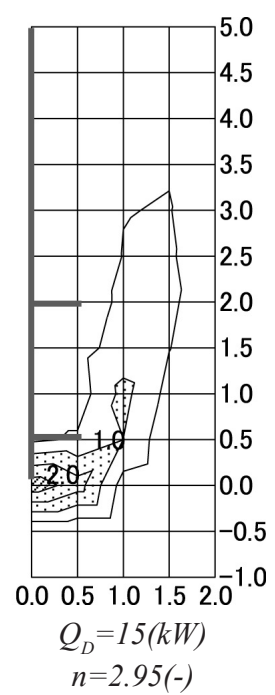

Wide Eaves

Window Shape $0.6(B) \times 0.3(H)(m)$

Figure 9(b) Non-dimentional Isotherms (Middle Scale Experiment)

It is considered that the non-dimensional temperature is independent of size and fire temperature but is uniquely determined only by gemetrical conditions, if window jet aspect ratio, $n$, were the same. That is, it is possible to predict real scale window jet plume temperatures based on the results of reduced scale experiments. 
If the temperatures at the locations of interest, $\Delta T(x, y, z)$, are measured in a reduced scale experiment, the non-dimensional temperature $\Theta\left(x^{*}, y^{*}, z^{*}\right)$ can be established as discussed in the avove. Then, the temperatures at geometrically similar locations in real scale building can be obtained since $\Theta\left(x^{*}, y^{*}, z^{*}\right)$ is the same between the reduced scale and the real scale as long as the window jet geometry is similar.

\section{TEMPERATURE IN THE VICINITY OF WINDOW PANEL IN WALL HAVING NO EAVES}

The window jet plume temperature is dependent on window geometry and arrangement of eaves above a window. Therefore, in general, it is necessary to predict real scale window jet plume temperatures based on the results of reduced scale experiments. However, for the cases where there are no eaves above window, it is convenient to establish the non-dimensional temperature of the window jet plume in the vicinity of window panel in advance based on appropriate reduced scale experiments since this is the case we often encounter in practical building external wall design.

Here, the experiments conducted for establishing the non-dimensional temperature are presented and the assessment method of the temperature in the vicinity of window panel in no eaves is proposed.

\subsection{Measurement condition of small scale experiments}

This series experiment was performed using the small-scale setup shown in Figure 2. The conditions of the windows and fire sources in the small-scale experiments are shown in Table 2. In addition, in experiment No.6 and No.7, side walls were installed on the both sides of the window as shown in Figure 10. The side wall is for restricting the air entrainment from the side of the window jet plume so that the window can be considered to be equivalent to infinitely wide window. The side wall was set slightly inside the window side in order to avoid the influence of window jet contraction. 
Table 2 Conditions for Small Scale Experiment

\begin{tabular}{|c|c|c|c|c|}
\hline \multirow{2}{*}{ Exp. No. } & \multicolumn{2}{|c|}{ Window Shape } & \multirow{2}{*}{ Side Wall } & \multirow{2}{*}{$\frac{\text { Fire Source }}{\text { Diameter }(\mathrm{m})}$} \\
\hline & Width B(m) & Height H(m) & & \\
\hline $1-1$ & \multirow{3}{*}{0.10} & \multirow{3}{*}{0.20} & \multirow{3}{*}{-} & 0.10 \\
\hline $1-2$ & & & & 0.15 \\
\hline $1-3$ & & & & 0.20 \\
\hline $2-1$ & \multirow{3}{*}{0.15} & \multirow{3}{*}{0.20} & \multirow{3}{*}{ - } & 0.10 \\
\hline $2-2$ & & & & 0.15 \\
\hline $2-3$ & & & & 0.20 \\
\hline $3-1$ & \multirow{3}{*}{0.20} & \multirow{3}{*}{0.20} & \multirow{3}{*}{-} & 0.10 \\
\hline $3-2$ & & & & 0.15 \\
\hline $3-3$ & & & & 0.20 \\
\hline $4-1$ & \multirow{3}{*}{0.20} & \multirow{3}{*}{0.15} & \multirow{3}{*}{-} & 0.10 \\
\hline $4-2$ & & & & 0.15 \\
\hline 4-3 & & & & 0.20 \\
\hline $5-1$ & \multirow{3}{*}{0.20} & \multirow{3}{*}{0.10} & \multirow{3}{*}{-} & 0.10 \\
\hline $5-2$ & & & & 0.15 \\
\hline $5-3$ & & & & 0.20 \\
\hline $6-1$ & \multirow{3}{*}{0.20} & \multirow{3}{*}{0.15} & \multirow{3}{*}{$\mathrm{D}=0.15(\mathrm{~m})$} & 0.10 \\
\hline $6-2$ & & & & 0.15 \\
\hline $6-3$ & & & & 0.20 \\
\hline $7-1$ & \multirow{3}{*}{0.20} & \multirow{3}{*}{0.10} & \multirow{3}{*}{$\mathrm{D}=0.15(\mathrm{~m})$} & 0.10 \\
\hline $7-2$ & & & & 0.15 \\
\hline $7-3$ & & & & 0.20 \\
\hline
\end{tabular}

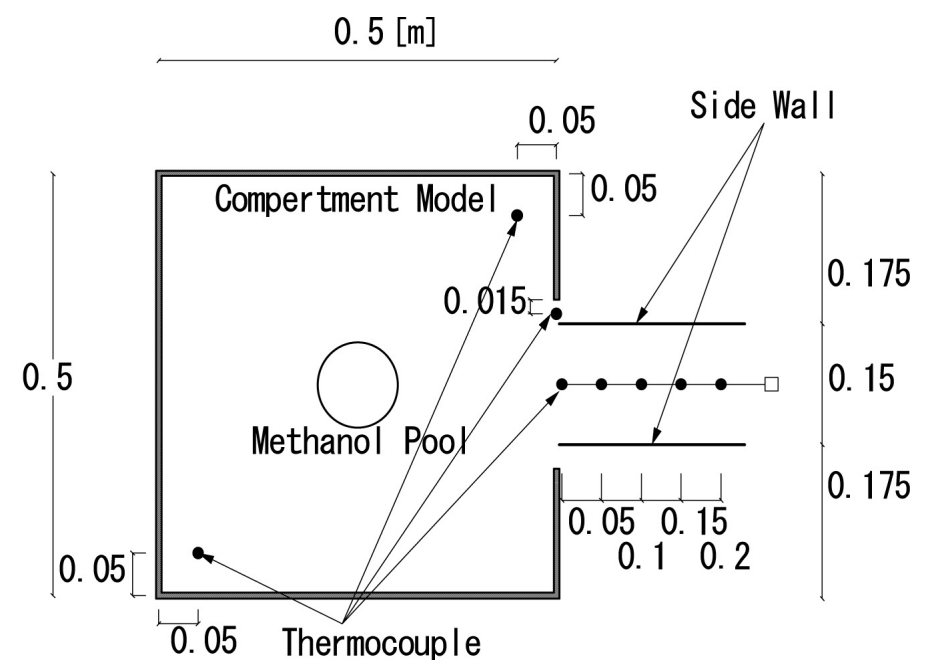

Figure 10 Schematic Diagram of Experimental Apparatus 


\subsection{Temperature of the window jet plume in the vicinity of window panel}

In order to calculate the non-dimensional temperature $\Theta\left(x^{*}, y^{*}, z^{*}\right)$, the non-dimensional heat flow rate of window jet $Q_{D}^{*}$ need to be estimated. The non-dimensional heat flow rate of window jet $Q_{D}^{*}$ is given by Equation (5) or Equation (7). However, in this study, Equation (7) which can take the factor of the window width into consideration is used as quantity of the non-dimensional heat flow rate of the window jet. In addition, the ejection height of window jet $H-Z_{n}$ is used instead of the height of window $H$, i. e.

with

$$
Q_{D\left(H-Z_{n}\right)}^{\prime^{*}}=\frac{Q_{D}}{C_{p} \rho_{\infty} T_{\infty} \sqrt{g} B\left(H-Z_{n}\right)^{3 / 2}}
$$

$$
\left(x^{*}, y^{*}, z^{*}\right)=\left(\frac{x}{H-Z_{n}}, \frac{y}{H-Z_{n}}, \frac{z}{H-Z_{n}}\right)
$$

Equation (10) is a slight modification of Equation (7), in which $H-Z_{n}$ is used instead of for the purpose of calculating non-dimensional temperature based on more accurate window jet geometry.

Then $n$ indicates the aspect ratio of the window jet, defined as

$$
n \equiv B /\left(H-Z_{n}\right)
$$

In addition, in experiment No.6 and No.7 with side walls, the aspect ratio of the window jet, defined as

$$
n^{\prime} \equiv D /\left(H-Z_{n}\right)
$$

where $D$ is the interval distance of side wall.

\subsection{Non-dimensional Temperatures}

Figure 11 shows the non-dimensional temperatures $\Theta\left(0,0, z^{*}\right)$ processed by Equation (4) using the non-dimensional heat flow rate defined by Equation (10).

In order to check the similarity of the non-dimensional temperature in the conditions that the aspect ratio is larger, the result of the full scale experiments were added for reference [6,7]. The full-scale experiment conditions are shown in Table 3.

Shown in Figure 11, it can be said that the non-dimensional temperature increase as the aspect ratio of the window jet became large.

Table 3 Conditions for Full Scale Experiment

\begin{tabular}{c|c|c|c|c}
\hline \multirow{2}{*}{ Exp. No. } & \multicolumn{2}{|c|}{ Window Shape } & \multirow{2}{*}{ Side Wall } & $\begin{array}{c}\text { Fire Source Heat } \\
\text { Release Rate }(\mathrm{kW})\end{array}$ \\
\cline { 2 - 3 } & Width B(m) & Height H(m) & & 500 \\
\hline \hline full scale-1 & \multirow{2}{*}{2.44} & \multirow{2}{*}{1.88} & - & 700 \\
\hline full scale-2 & & & & 900 \\
\hline full scale-3 & & & & \\
\hline
\end{tabular}




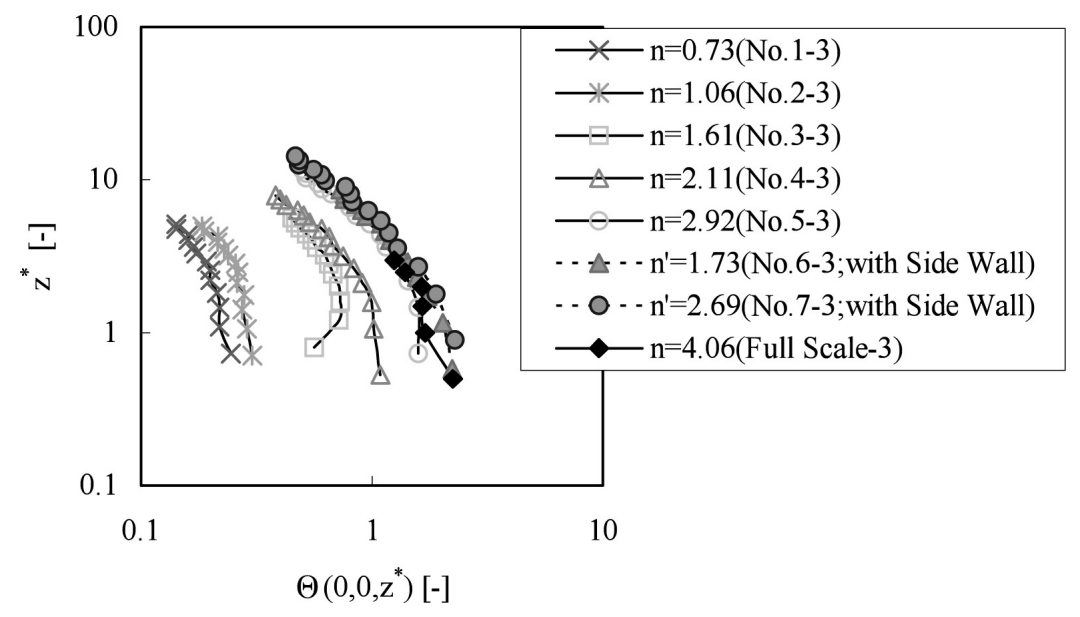

Figure 11 Non-dimensional Temperature

Next, the relationship of the non-dimensional temperatures $\Theta$ with the non-dimensional height $z^{*}$ are investigated by assuming as,

$$
\Theta\left(0,0, z^{*}\right)=\alpha z^{* \beta}
$$

Figure 12 shows the relation between the non-dimensional height $z^{*}$ and $\beta$. In the area that the non-dimensional height, $z^{*}$, is lager than about 2.0, the non-dimensional temperatures are negatively proportional to $-3 / 5$ power of non-dimensional height, i. e. $\Theta\left(0,0, z^{*}\right) \propto z^{*-3 / 5}$. On the other hands, in the area that the non-dimensional height, $z^{*}$, is less than 2.0, the non-dimensional temperatures are not significantly dependent on of the non-dimensional height, and may be regarded about constant.

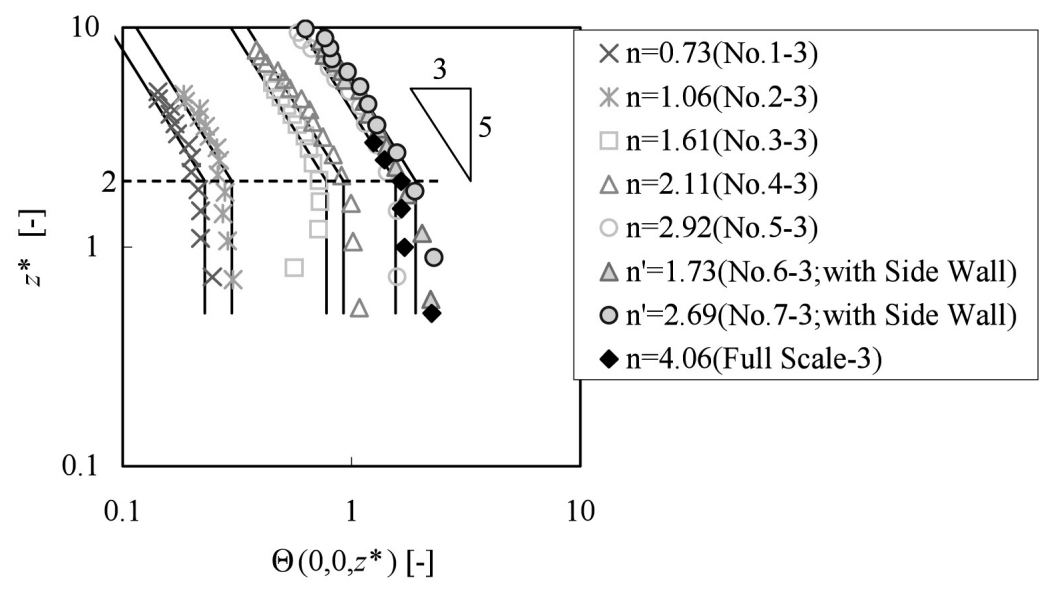

Figure 12 The Relation Between $z^{*}$ and $\beta$

Figure 13 shows the examples of the relation between the non-dimensional height to the power of $-3 / 5$, i. e. $z^{*-3 / 5}$, for the various aspect ratio of the window jets and the non-dimensional temperature, $\Theta\left(0,0, z^{*}\right)$. The inclination of the straight line show $1 / \alpha$. 


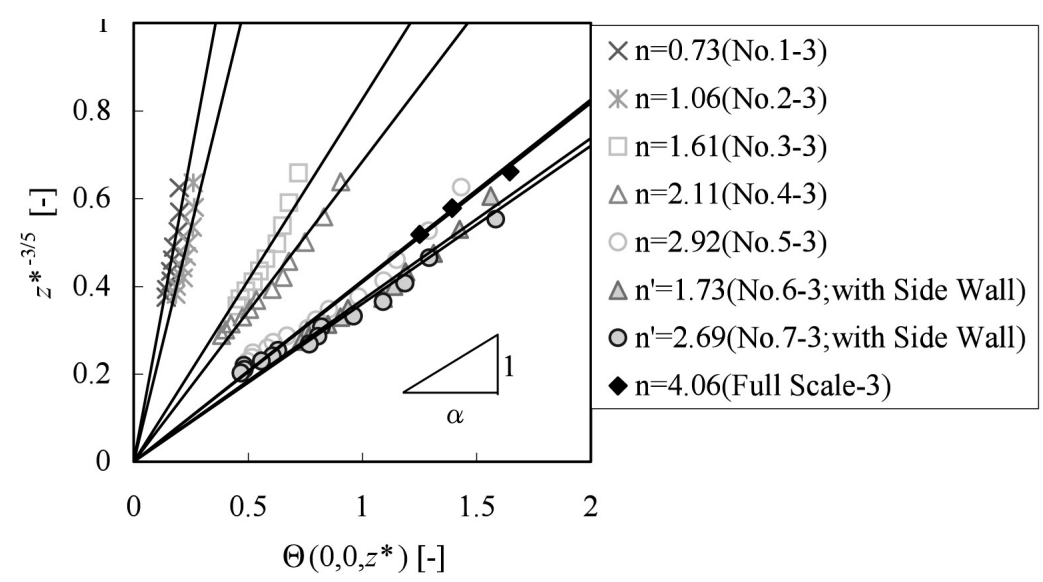

Figure 13 The Relation Between $z^{*-3 / 5}$ and $\Theta\left(0,0, z^{*}\right)$

Figure 14 shows the relation between $\alpha$ and the aspect ratio $n$ obtained from the all experiment results, in which the values of $\alpha$ were plotted v. s. the aspect ratio $n$. Remind, the No.6 and No.7 are the cases that side walls are attached to the window. As shown in Figure 14, the value of $\alpha$ increases with aspect ratio up to $n=3.3$, but does not increase any more for $n>3.3$, at which $\alpha$ takes the maximum value of 2.7. The side wall cases also takes $\alpha=2.7$.

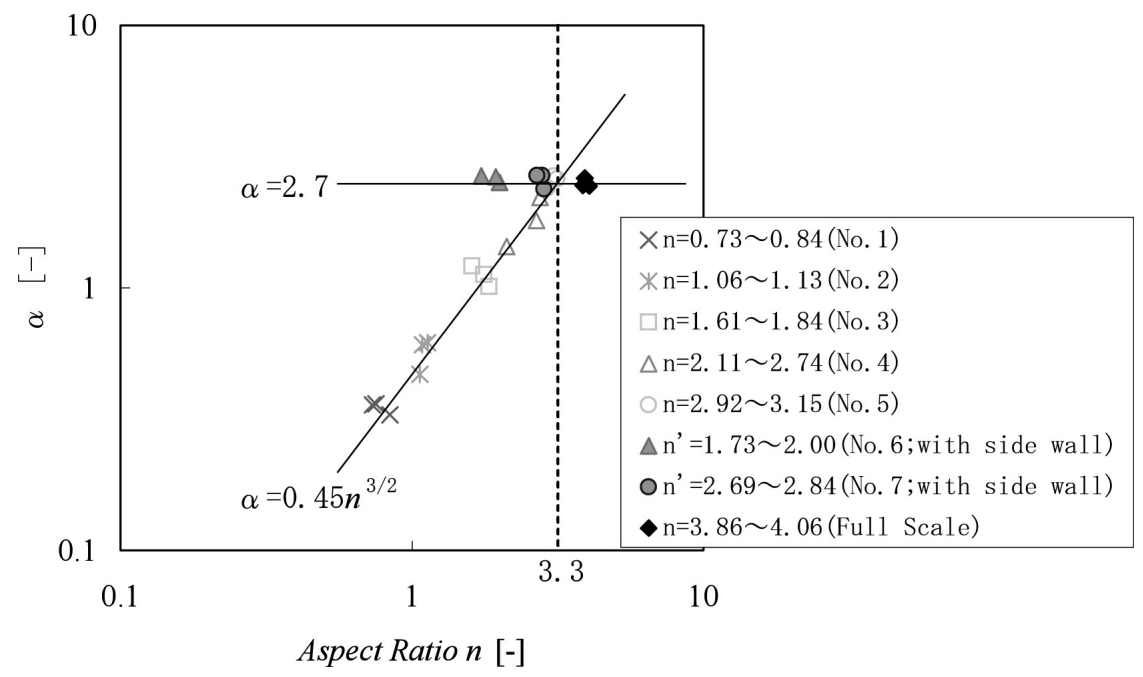

Figure 14 The Relation Between $\alpha$ and $n$

Based on the above, the non-dimensional temperatures in the non-dimensional height for $z^{*} \geq 2$ can be proposed as follws,

$$
\begin{aligned}
& \Theta\left(0,0, z^{*}\right)=\alpha z^{*-3 / 5} \\
& \alpha=\left\{\begin{array}{cc}
0.45 n^{3 / 2} & (n \leq 3.3) \\
2.7 & (3.3<n)
\end{array}\right.
\end{aligned}
$$


In the region where the non-dimensional height is smaller than 2 , i. e. $z^{*}<2$, where the dependence of non-dimensional temperature $\Theta\left(0,0, z^{*}\right)$ on $z^{*}$, substituting $z^{*}=2$ in Equation (15) gives,

$$
\Theta\left(0,0, z^{*}\right)=0.66 \alpha
$$

Figure 15 plots the all experimental results of the relation between the non-dimensional height, $z^{*}$, and the non-dimensional temperatures which is divided into $\alpha$, i.e. $\Theta\left(0,0, z^{*}\right) / \alpha$.

As shown in Figure 15, it is demonstrated that the calculated non-dimensional temperatures agree well with the measured non-dimensional temperatures.

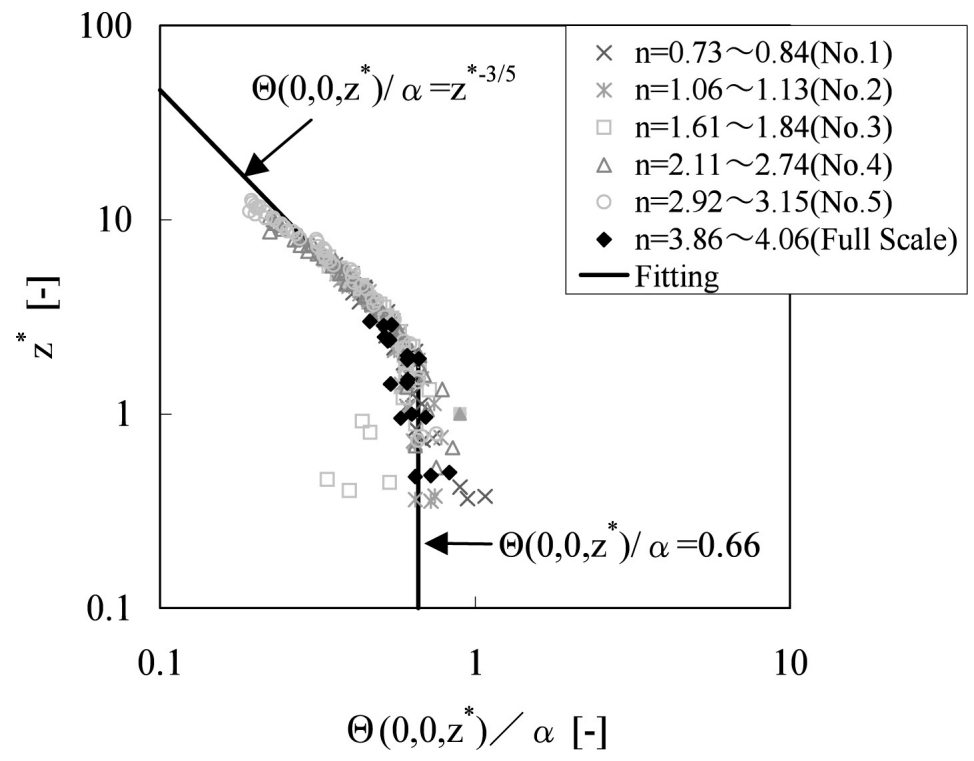

Figure 15 Comparision of Measured Non-dimensional Temperatures and Calculated Non-dimensional Temperatures

\subsection{Calculation procedure the Temperature of the window jet plume}

For non-eaves configuration, the temperature of the window jet plume on the surface of a window can be evaluated by the calculation procedure, step 1 - step8, as follows:

(Step1) Calculate the fire room temperature $T_{s}$

(Step2) Calculate the neutral plane at the window $Z_{n}$

(Step3) Calculate the aspect ratio $n$ by Equation (12)

(Step4) Calculate the non-dimensional height $z^{*}$ by Equation (11)

(Step5) Calculate the $\alpha$ by Equation (16)

(Step6) Calculate the non-dimensional temperature $\Theta\left(0,0, z^{*}\right)$ by Equation (15) or Equation (17)

(Step7) Calculate the non-dimensional heat flow rate $Q_{D\left(H-Z_{n}\right)}^{\prime *}$ by Equation (10)

(Step8) Calculate the temperature of the window jet plume $\Delta T(0,0, z)$ by Equation (4) 


\section{CONCLUSION}

It was found that the non-dimensional temperature is independent of size and fire temperature but is uniquely determined only by geometrical conditions, that is, it is possible to predict real scale window jet plume temperatures based on the results of reduced scale experiments.

In this study, in order to develop a means to assess the effectiveness of some design elements for preventing upper floor fire spread, feasibility of scaling of window jet plume temperature distribution by means of a nondimensional temperature was explored. From the results obtained through small scale and middle scale experiments, the following findings were obtained: (1) the non-dimensional temperature is independent of size and fire temperature but is uniquely determined only by geometrical conditions, (2) the calculating the temperatures of window jet plume in arbitrary positions, i.e. $\Delta T(x, y, z)$, the non-dimension temperature $\Theta\left(x^{*}, y^{*}, z^{*}\right)$ can be established by the reduced scale experiments instead of the process from step6 to step8.

For the particular case where there is no eaves or balcony above a window, it is found that the non-dimensional temperature at the vicinity of upper floor window pane is proportionate to the aspect ratio of window jet section and the temperature evaluation method was developed based on the analyses of the results of the reduced scale experiments.

\section{REFERENCES}

1. Yokoi, S. : Study on the Prevention of Fire-Spread Caused by Hot Upward Current, Report of The Building Research Institute, BRI, 1960.

2. Thomas, P.H. and Law, M. : The projection of flames from burning buildings, Fire Research Note, No.921, 1972

3. Ohmiya, y., and Hori, y. : Properties of external flame taking into consideration excess fuel gas ejected from fire compartment, Journal of Archit. Plann. Environ. Engng. , No. 545, p.1, 2001. (in Japanese)

4. Yamada, T., Kurioka, H., Yanai, E., Suzuki, T., Abe, N., Iida, A., Sekizawa, A., Satoh, H. and Shinohara, M. : Modeling of Ejected Flames through Openings and Case Study Examples, Summary of papers annual meeting of Japan Association for Fire Science and Engineering, pp.128-131, 2004.5. (in Japanese)

5. Yamaguchi, J., Yamada, S., Tanaka, T. and Wakamatsu, T.: Measurement for Mass Flow Rate of Door Jet in Compartment Fire, Transaction of AIJ, No. 501, pp.1-7, 1997. (in Japanese)

6. Sagimori, K., Ito, A., Yamaguchi, J., Iwai, Y., Harada, K., Tanaka, T. and Wakamatsu, T.: Scaling Law of Temperature Profile in Window Jet Plume, AIJ Annual Conference, pp.235-236, 1998.9. (in Japanese) 
7. Ito, A., Sagimori, K., Yamaguchi, J., Iwai, Y., Harada, K., Tanaka, T. and Wakamatsu, T.: Temperature Prediction of Window Jet Plume, AIJ Annual Conference, pp.237-238, 1998.9. (in Japanese) 